\title{
Challenges for long-lived massive particle searches at the LHC
}

\author{
Andrea GIAMMANCO*† \\ Université Catholique de Louvain, Center for Particle Physics and Phenomenology, \\ Louvain-la-Neuve (Belgium) \\ E-mail: andrea.giammanco@cern.ch
}

The most recent studies by the CMS and ATLAS collaborations are presented on the sensitivity to searches for long-lived massive particles. Such particles appear in several extensions of the Standard Model and are allowed by the present bounds coming from cosmology and existing accelerator data. We show the challenges to event triggering and reconstruction posed by such particles, and how CMS and ATLAS plan to deal with them. Particular emphasis is given to possible early discoveries, i.e., with $100 \mathrm{pb}^{-1}$ or less. In special cases, long-lived particles may come to rest in dense material, and decay at much later times; these can be searched for by triggering during periods with no beam-beam collisions.

European Physical Society Europhysics Conference on High Energy Physics July 16-22, 2009

Krakow, Poland

\footnotetext{
*Speaker.

On behalf of the CMS and ATLAS collaborations.
} 


\section{Introduction}

Both CMS [1] and ATLAS [2] are general-purpose $4 \pi$ detectors, each of them composed by several sub-systems, with a relatively high degree of complementarity between their designs. These facts allow a wide range of signatures to be explored at the LHC, including some very exotic ones.

Here we consider a few examples of the class of signatures involving an exotic particle able to traverse macroscopic distances before decaying. Long-lived or stable states could exist in several extensions of the Standard Model; to name a few examples, in some supersymmetric models like the Gauge Mediated Supersymmetry Breaking (GMSB) [3], in SplitSUSY [4] due to the large gluino/squark mass splitting, or in "hidden valley" scenarios due to a potential barrier [5]. Several more examples are considered in Ref. [6]. Long but finite lifetimes are especially interesting in cosmology since this would affect the primordial light element abundances and could solve the discrepancy between the observed ${ }^{6} \mathrm{Li},{ }^{7} \mathrm{Li}$ abundances and standard big bang nucleosynthesis calculations $[7,8]$.

This report follows a signature-oriented approach, by focusing on the experimental challenges posed by these unconventional final states and the strategies devised by the CMS and ATLAS collaborations to overcome them. Section 2 deals with the case of such a particle being charged and leaving an observable track in the detector; Section 3 considers the case of a long-lived particle being stopped in the detector volume and decaying after a macroscopical time delay; Section 4 explores the signature of neutral long-lived particles decaying into energetic photons.

\section{Charged exotic particles traversing the detector before decaying}

The main feature of these particles, from the experimental point of view, is their slowness. When the particle is not sensitive to the strong interaction (as in the cases of charginos, staus, Kaluza-Klein resonances of leptons) it completely traverses the detector volume and it is seen as a "slow muon". In some models the long-lived particles are sensitive to the strong interaction (e.g., gluinos or squarks) and therefore form the so called R-hadrons [9], by bounding with light quarks which neutralize the colour quantum number; this has an huge impact on their phenomenology, since in traversing the detector there is a high probability of nuclear interactions, which can change the light quark content of the R-hadron and thus occasionally change its electric charge during the traversal.

One of the main bottlenecks, when searching for these particles, is the trigger efficiency, which is limited by the fact that particles with $\beta<0.6$ need more than one bunch crossing time (25 ns) to reach the muon chambers; in the R-hadrons case, additionally, charge flipping reduces the efficiency of track reconstruction in the trigger algorithm. In CMS, the trigger path based on the presence of a standard muon-like particle has an efficiency of 75 - 97\% (depending on the mass and momentum spectra predicted by the model) for colour-neutral long-lived exotics, and much lower (11 - 24\%) for R-hadrons. Triggers based on the transverse missing energy (MET) of the event are more efficient for R-hadrons and do not suffer from the bunch-crossing assignment issue, thus resulting complementary to the muonic paths. On the other hand, the missing energy measurement is affected by the topology of the signal event and the MET triggers efficiencies are therefore much more model dependent. 
The ATLAS collaboration attacked the problem by designing a dedicated trigger at Level 2 based on the time of flight in the muon system [11], where the RPCs have a time resolution of $3 \mathrm{~ns}$, recovering hits assigned to other bunch crossings by the Level 1 step. This trigger path doesn't require any matching between the object reconstructed in the muon chambers and in the inner tracking system, therefore it doesn't loose efficiency for charge-flipping particles. With this dedicated trigger, the efficiency for a stable $300 \mathrm{GeV}$ gluino increases from $39 \%$ to $92 \%$.

In addition, ATLAS studied an offline topological selection expressely designed for the Rhadron case: two "muon-like" tracks from the muon system are matched to the inner tracking system allowing for the sign of the charge to be opposite in the two parts of the detector; their directions are required to be almost back to back; since slow massive particles release much energy by ionization, the information from the Transition Radiation Tracker of the inner detector is exploited by taking the ratio of hits above the high $(6.5 \mathrm{keV})$ and the low $(200 \mathrm{eV})$ thresholds; since the R-hadron fragmentation is a very asymmetric one, few tracks and no hard jet are allowed in their proximity. An intriguing possibility is to be able to distinguish gluinos from stops (or other squarks) by the evidence or not of charge flipping between inner detector and muon system: the exchange of light quarks in a single nuclear interaction can lead to a full charge inversion only in the gluino case, while charged R-hadrons containing stops can only become neutral.

The CMS collaboration follows a model-independent approach [10], by looking at variables which only depend on the speed $\beta$ of the particle: the specific ionization $(d E / d x)$ in the Silicon Strip Tracker (SST) and the time of flight as measured in the Drift Tubes (DT) of the external muon system.

Very early observation (of the order of $100 \mathrm{pb}^{-1}$ of integrated luminosity) is possible for sub$\mathrm{TeV}$ masses, in models where the production is mediated by the strong force.

\section{Stopped exotic particles decaying in the calorimeters}

R-hadrons, due to their additional nuclear interactions, can loose enough energy in traversing matter to stop inside the detector, most probably inside the calorimeters. These particles must eventually decay, leading to a striking signature independent from the presence of beam. The CMS collaboration [12] devised a specific trigger, requiring for an energetic jet in anti-coincidence with the beam monitors, to be run both during normal data-taking periods, in order to profit from the gaps in the beam time structure (and be thus sensitive to short R-hadron lifetimes), and in the "interfill periods", i.e., after a fill has been dumped, and even during longer breaks in the accelerator activity while the detector is taking cosmic and commissioning data.

The only possible background sources for such a search are calorimeter noise, detector faults, and cosmic-ray induced hadrons. All these have already started to be studied during the cosmic data-taking campaigns [13], and both the trigger and off-line selections for this search are optimized by modeling the background over this certainly signal-free sample. As a benchmark, models where the long-lived particle is a gluino are being considered, although the search applies to any strongly produced exotic.

This search is in principle sensitive to gluino lifetimes ranging from hundreds of nanoseconds to months. Assuming a production cross section of $0.5 \mathrm{nb}$, as expected for the strong production of $m_{\tilde{g}}=300 \mathrm{GeV}$ gluinos, an instantaneous LHC luminosity of $10^{32} \mathrm{~cm}^{-2} \mathrm{~s}^{-1}$ at $10 \mathrm{TeV}$ would 
already give sensitivity to lifetimes ranging from microseconds to weeks, within a few days of operation.

\section{Non-pointing photons}

The primary motivation to look for energetic photons not coming from the interaction vertex is provided by GMSB [3], although the same signature can be present in other models, e.g., "hidden valley" scenarios [5]. GMSB is a spontaneously broken local supersymmetry. It can manifest itself at the LHC through the production of strongly interacting squarks and gluinos yielding long decay chains, leading to final states with many leptons and jets. Its main phenomenological feature is related to the identity of the Lightest Supersymmetric Particle (LSP), which is a Gravitino with very low mass (less than a few KeV's), while the neutralino or the stau is the Next-to-Lightest Supersymmetric Particle (NLSP). Given the weakness of the coupling with the Gravitino, the lifetime can be very long. The case where the stau is a long-lived NLSP is within the scope of Sec. 2. If the neutralino is a NLSP, it can decay to a Gravitino and a photon, and it is possible to have photons emitted far from the interaction point.

A study from the ATLAS collaboration [11] adds to the standard search for GMSB, based on counting the numbers of hard photons and of opposite-sign same-flavour leptons, a neutralino lifetime measurement based on the timing of the photon arrival on the electromagnetic calorimeter and on the distance between the baricenters of the clusters recorded in the front layer and the middle layer of the calorimeter.

Differently from ATLAS, the electromagnetic calorimeter in CMS is not longitudinally segmented, therefore the corresponding CMS study [14] is based on the shape of the electromagnetic shower in the $\eta-\phi$ plane, which is typically circular for photons coming from the center of the detector while it is a tilted ellipse in the case of non-pointing photons.

A discovery is feasible with a few inverse femtobarns over a large range of parameters.

\section{Conclusions}

Long-lived exotic particles have non-standard signatures which open parameter space regions inaccessible to standard analyses. Their searches often require a use of the detector information that was not foreseen at the time when the LHC experiments were being designed. The CMS and ATLAS detectors are mostly complementary, thanks to widely different design choices in several of their sub-systems.

A common feature of the signatures described in this report is that their background is mostly of instrumental origin. On the other hand, this means that a very deep knowledge and control of the detectors is needed in order to perform the search, with the consequence that a strong link exists between the high-level analyses of this kind and the low-level commissioning activities. The already taken and still ongoing "cosmic runs" are valuable data for understanding the detectors to the high precision needed for these exotic searches, and even more so in cases where the cosmic particles themselves are estimated to be one of the main components of the background. 


\section{References}

[1] CMS Collaboration, JINST 3:S08004,2008

[2] ATLAS collaboration, JINST 3:S08003,2008

[3] L. Alvarez-Gaumé, et al., Nucl.Phys. B207 (1982) 96-110

[4] N. Arkani-Hamed and S. Dimopoulos, JHEP 06, 073 (2005)

[5] M. Strassler and K. Zurek, Phys.Lett. B651, 374 (2007)

[6] M. Fairbairn, et al., Phys.Rept. 438, 1-63 (2007)

[7] A. Arvanitaki, et al., Phys.Rev. D79 (2009) 105022

[8] T. Jittoh, et al., Phys.Rev.D78 (2008) 055007

[9] R. Mackeprang and A. Rizzi, Eur.Phys.J. C50 (2007) 353-362

[10] CMS collaboration, EXO-08-003

[11] ATLAS collaboration, CERN-OPEN-2008-020

[12] CMS collaboration, EXO-09-001

[13] CMS collaboration, CFT-09-008

[14] L. Goscilo, et al., CMS Analysis Note 2006/095 